\title{
INICIACIÓN Y FORMACIÓN DEPORTIVA: UNA REFLEXIÓN SIEMPRE OPORTUNA
}

\section{INITIATION AND SPORTS EDUCATION: A REFLECTION ALWAYS ON TIME}

\author{
Edgar Raúl Acosta1
}

${ }^{1}$ Lic. Educación Física, c. Magister en intervención integral al deportista de la Universidad Autónoma de Manizales, Especialista en Bio - metodología del Entrenamiento Deportivo, Especialista en Ejercicio Físico para la salud, Especialista en Pedagogía. Docente universitario U.D.C.A Email: eacosta@udca.edu.co

Rev. U.D.C.A Act. \& Div. Cient. 15 (Supl. Olimpismo): 57 - 65, 2012

\section{RESUMEN}

Ampliamente se encuentra documentado y desde hace varias décadas, que la iniciación deportiva, entendida como un proceso multifacético, es la base para el desarrollo deportivo de un país, pero más importante aun, para la formación de ciudadanos físicamente activos y con altos valores de convivencia social y ambiental. Este proceso como tal, no es bien conocido y comprendido no solo por la mayoría de personas sino por los dirigentes deportivos, los políticos, los comunicadores y la mayoría de profesionales quienes trabajan en el área del deporte, generando procesos sin coherencia ni continuidad, que garanticen procesos deportivos y de convivencia socio-ambiental. Es imperativa la revisión teórica y de prácticas sobre la iniciación y la formación deportiva que se realiza en la actualidad, para fortalecer políticas claras, a nivel nacional, que permitan la consolidación de la cultura deportiva nacional.

Palabras clave: Deporte, iniciación y formación deportiva.

\section{SUMMARY}

For decades the fact, that the sports initiation, understood as a multifaceted process, is the basis for a country's sports development, has been widely documented. However, it is still more important to fulfill the objective of forming physically active citizens with high values of social and environmental coexistence. This process is not well known and understood, not only by most people, but also by sport leaders, politicians, journalists and many sport professionals. This fact origins that many of the developed processes in this area lack the coherence and continuity, necessary to guarantee the scope of sporting triumphs and of social and environmental harmony. Therefore it becomes imperative to carry out a theoretical and practical revision of the actual practices on initiation and sports training, in order to strengthen clear politics at the national level which enables the consolidation of a national sports culture.

Key words: Sport, initiation and sports training.

\section{INTRODUCCIÓN}

En el año de los juegos Olímpicos, periodistas y comunicadores, políticos, dirigentes deportivos y la gente, en general, hablan y pronostican sobre los resultados de la participación de los deportistas, quienes representarán a su país. Pocos saben que todo inicia con un proceso organizado e ininterrumpido desde edades muy tempranas, que asegure el mejor desempeño de acuerdo a las posibilidades. El concepto de deporte es dinámico y cambiante de acuerdo a la cultura de cada país o región, pero los colombianos que trabajan en este campo, deben tener una idea de lo que significa el deporte, pues se ha convertido en uno de los fenómenos más influyentes, en la historia contemporánea de Colombia, puesto que involucra un amplio campo de conocimientos y de ciencias aplicadas, para su comprensión y su expresión.

Todo aquel que tenga relación directa o indirecta con esta manifestación social debe profundizar en los estudios de su génesis, su evolución y sus diversas manifestaciones en la multiplicidad de contextos, en los cuales, tienen sus expresiones. Dada la amplitud e importancia de la temática para el país en aspectos no solo deportivo, los son también los de índole social, ambiental, político y económico, entre otros. Los directos responsables, los docentes de educación física, los entrenadores de iniciación, de selección y de altos logros y hasta los dirigentes de alto nivel, deben tener claros los conceptos básicos que le permitan delimitar el concepto 
deporte, iniciación y formación deportiva, para la aplicación de políticas y de programas sostenibles en el tiempo, con resultados que se puedan pronosticar.

El objetivo del presente manuscrito es convocar a la reflexión, a partir de una revisión de los conceptos y las prácticas sobre la iniciación y la formación deportiva, a todos los implicados en este proceso

\section{MATERIALES Y MÉTODOS}

El presente ejercicio investigativo, se basa en la metodología de tipo exploratorio-descriptivo, explicada por Hernández et al. (2007), que responde a diversas características, como revisar una temática, sobre la cual, se presentan dudas, para aclarar e indagar nuevas perspectivas o ampliar las existentes. La problemática a abordar es particular al deporte nacional y pretende evaluar la posibilidad de constituirse como línea de investigación al interior de la Facultad de Ciencias de Deporte de la Universidad de Ciencias Aplicadas y Ambientales U.D.C.A.

Diseño de investigación: El ejercicio de investigación, se realizó bajo las características de diseño de investigación no experimental longitudinal, que analiza los cambios, a través del tiempo de un evento, una comunidad, un fenómeno, una situación o un contexto (Hernández et al. 2006). Cumple con la característica de describir y de reflexionar sobre los cambios, por medio del tiempo de la categoría, deporte e iniciación y formación deportiva.

Se realizó una revisión de la literatura con el siguiente protocolo: 1) Determinar las palabas clave para la búsqueda en bases de datos en internet (Medline - PubMed (National Library of Medicine), Cochrane data base of systematic reviews, Lilacs/Bireme, Doyma, Scielo, Biblioteca Virtual de Salud, ProQuest) y buscadores (Google académico y Mozilla Firefox), páginas Web de entes nacionales e internacionales de la temática investigada, fuentes de información, como bibliotecas (U.D.C.A y Virgilio Barco); 2) Se determinaron criterios de inclusión y de exclusión de documentos; 3) Se evaluó la pertinencia y la calidad de los documentos y, 4) La unidad de análisis serán los documentos que incluyan investigaciones y revisiones teóricas sobre conceptos de deportes e iniciación deportiva.

\section{RESULTADOS Y DISCUSIÓN}

Sobre la palabra "deporte", Koch (1981) describe que provine del verbo latino "deportarte", distraerse y, luego, del sustantivo francés e inglés, en la forma "desport" o "disport", es decir, diversión. El deporte es un término que se usa indife- rentemente para referirse a muchas actividades, donde expresiones sociales se encuentran mediadas por el movimiento humano, el ejercicio físico, la competencia, la recreación, la motricidad y la lúdica, entre otros. En manifestaciones deportivas, como los Juegos Olímpicos o los campeonatos del mundo en diferentes disciplinas, se habla de deporte; pero también se utiliza el término para expresar otras actividades, como los campeonatos al interior de una empresa, de una escuela o las actividades de fin de semana en los parques de la ciudad. Para no caer en equivocaciones, el concepto de deporte debe estar contextualizado desde aspectos sociales, temporales, económicos, políticos y educativos, que permitan la construcción del hecho social, sin caer en contradicciones. Para Cagigal (1975, 1990), se deben contemplar tres elementos fundamentales, para que el deporte se manifieste en cualquier sociedad: el juego, la competición y el ejercicio físico y contar con algunas funciones, a partir de aspectos sociales, como el ocio, el esparcimiento, la higiene, el desarrollo biológico, la salud, las relaciones sociales y la transmisión de valores.

El deporte actual tiene su origen y su evolución en Inglaterra, siglos XVIII y XIX, en donde confluyeron factores fundamentales para su génesis, que según Elías \& Dunning (1992), estos fueron: el verano londinense, donde se reunían las ciudadanos, de origen rural y urbano, a compartir actividades de sus regiones; luego, éstas se combinaron, se reglaron y se trasformaron en otras, como el criquet y el boxeo. Este mismo autor comenta sobre el deleite que manifestaban los ingleses para reunirse o agruparse, según la actividad o agrado por ciertas dinámicas deportivas y es, precisamente, donde surgen los "clubs", que se convierten en una de las expresiones propia de los caballeros londinenses: se conforman clubes de caza y de juegos de pelota, entre los más significativos, que favorecieron el desarrollo del deporte moderno, con la inclusión y el respeto a los reglamentos. "Cuando surgió la costumbre de organizar competiciones por encima del nivel local dado que los equipos de críquet viajaban de un lugar a otro, hubo que garantizar la uniformidad del juego" (Elías E Dunning, 1992). De esta manera, se consolida la reglamentación de actividades, que son el comienzo de los deportes modernos.

Otro elemento importante para la gestación del deporte moderno son las escuelas y el sistema escolar (Velásquez, 2001). Los estudiantes de las academias públicas practicaban los juegos tradicionales populares de campesinos, de artesanos y de comerciantes. "Estos juegos son violentos y por lo tanto, son prohibidos por el profesorado de los internados de secundaria y por las universidades" (Rodríguez, 2000); con el paso del tiempo, los pedagogos de estos establecimientos ven en las actividades físicas y regladas aspectos formativos, las incluyen en los currículos y le adicionan aspectos formativos, con propósitos educativos. 
Otro aspecto que se debe destacar en el surgimiento del deporte moderno es el planteado por Diem (1966), quien se refiere al aspecto de la personalidad de los ingleses: el respeto y la aceptación de las reglas, su gusto por la práctica de juegos reglados y físicos (espíritu deportivo) y la aceptación de competir bajo parámetros y normas libremente aceptadas. Para este autor, estos fueron los aspectos que contribuyeron a la génesis, a la trasformación y a la evolución del deporte moderno.

Es elemental reconocer los aportes de los autores citados, en cuanto a los factores que gestaron el deporte moderno, para la orientación de la iniciación y la formación deportiva, como también, el apego y el respeto a los principios y a los valores, sobre los cuales, se sustenta el deporte. Se puede concluir, que el deporte moderno se apoya en cuatro elementos fundamentales: a) la regulación de la violencia, a través del ejercicio físico; b) la integración y la reunión de personas con afinidad y con gustos por una actividad; c) la inclusión del deporte por parte de la escuela, para apoyo en la formación de valores y aspectos académicos y, d) la aceptación libre, además del respeto por las normas y la reglamentación de la competencia.

La génesis del deporte, desde la experiencia inglesa, provee la posibilidad de comprender el deporte moderno, pero definitivamente no es la única, puesto que cada país y región mantiene e integra sus expresiones culturales al fenómeno deportivo, para la convivencia, el uso del tiempo libre, la trasmisión de valores y las costumbres.

Los Juegos Olímpicos de la antigua Grecia y los juegos Olímpicos de la modernidad son también movimientos culturales muy importantes para la humanidad, de donde se puede comprender el deporte desde sus orígenes a las manifestaciones más actuales.

Son innumerables los estudios sobre el origen y la evolución de los deportes. El más reciente, publicado en el 2010, por Xavier Pujadas, La Metamorfosis del Deporte, involucra aspectos que abarcan desde el mito, la religión y los rituales a los modernos problemas políticos, económicos y de segregación racial y de género, en la actualidad. Los Juegos Olímpicos, por su trayectoria histórica e importancia, tienen un campo amplio de estudio en esta época, ya que involucra a diferentes ciencias y disciplinas de conocimiento, para su comprensión. El Comité Olímpico Internacional, por ejemplo, promueve estudios sobre el fenómeno del Olimpismo y en cada país donde existe un Comité, se institucionalizan cursos sobre los juegos en diferentes espacios y contextos; las Cátedras Olímpicas son otros modelos de conocimiento que se encuentran vinculados en varias universidades de diferentes países, que ofertan estudios científicos-sociales, para comprender y ampliar el fenómeno deportivo (COI, 2010).

La educación y la pedagogía tienen una participación muy importante para el desarrollo del deporte, respaldado por autores muy influyentes para la implementación de la pedagogía y la educación formal (Acosta, 2008). Desde la perspectiva educativa, la evolución del deporte, a finales del siglo XIX e inicio del XX, continúo ligada a la educación, apoyada en teorías de grandes pensadores para la educación y la educación física, como Rousseau, Pestalozzi, Fröeber, Montessori y Dewey y por la creación de más clubes, federaciones de diversas modalidades deportivas, además de campeonatos, a nivel local, regional, nacional e internacional.

El deporte nacional cuenta también con su historia. Contecha (1999) ubica los inicios de las prácticas deportivas, en el país, con el nacimiento de la República; los libertadores establecieron políticas para la organización de la educación a la luz de las pedagogías inglesa y francesa, haciendo énfasis en la educación física y militar, con el fin de evitar un posible contra-ataque español, con el ánimo de recuperar los territorios. La educación pública, se reglamenta, inicialmente, con la ley 92 de 1888, que obliga a los colegios públicos a la enseñanza de la gimnasia. Es posible citar esta fecha y esta ley como el nacimiento del deporte escolar en Colombia. El recorrido histórico realizado por Contecha (1999), explica cómo se consolidó el deporte nacional, además de realizar un recuento de la legislación y la reglamentación del deporte nacional, hasta la década del 90.

En la historia más reciente para el deporte colombiano fue la creación del Consejo Nacional de la Juventud y el Deporte COLDEPORTES, por decreto 2743 de 1968, que entre sus funciones tiene: "Elaborar junto con el Ministerio de Educación Nacional, los planes sobre educación física, Deporte, fomento y estímulo a las actividades de bienestar y recreación de la juventud, las asociaciones y movimientos juveniles y demás aspectos similares, promover y acometer actividades que se encaminen a difundir y estimular la Educación Física y los Deportes en todo el territorio nacional, directamente o en cooperación con los departamentos, y las organizaciones y asociaciones interesadas en el deporte" (artículo 9). Y es COLDEPORTES, que por medio de la contratación de profesionales, diseña y propone actividades para el desarrollo de deporte y la educación física.

Pero, actualmente, no basta la creación de organizaciones por parte del Estado o entidades privadas al servicio del deporte, para cumplir objetivos en el orden de lo deportivo y lo social y no alcanza la formulación de leyes o de decretos que involucren a diferentes actores de la sociedad si existen 
deficiencias en la interpretación del hecho deportivo y los objetivos que se pretenden.

\section{Aproximación al deporte contemporáneo.}

Para Parlebas (2001), el juego deportivo es una "situación motriz de enfrentamiento codificada, denominada juego o deporte por las instancias sociales. Un juego deportivo se define por su sistema de reglas que determinan su lógica interna" y son más los autores, desde la sociología y la antropología, que se han preocupado por tratar de conceptuar el deporte para su comprensión y práctica social. Para poder orientar los procesos de iniciación y de formación deportiva es imperativo que se identifique cuál tipo de deporte queremos para nuestra sociedad: ċa cuál se le prestará más atención, recursos y apoyo?, ćcuál es el rol del Estado, la educación y la sociedad en cada una de sus manifestaciones?

El deporte actual involucra varios aspectos de las sociedades modernas. El lograr distinguir el punto donde se piensa al deporte como una necesidad para la juventud en la construcción de un modelo de hombre a un espectáculo que genera un alto número de recursos e intereses económicos y políticos es fundamental para los directos involucrados. Su importancia en las sociedades contemporáneas hace que lleguen a él diferentes ciencias y disciplinas de conocimiento (social y científico), generando valiosos aportes y enriqueciendo el amplio campo del deporte y las ciencias aplicadas. La tecnología, por su parte, contribuye a los estudios para el diseño de campos deportivos, elementos y materiales, software de entrenamiento deportivo, modelos de predicción y aplicaciones informáticas, que mejoran las posibilidades física, técnicas y tácticas de deportistas, a todo nivel. Una rápida mirada al futuro hace pensar en un constante cambio y trasformación del deporte.

La organización del deporte actual clasifica a los deportistas dependiendo del objetivo a alcanzar. Quienes entrenan en busca de altos logros tienen el apoyo para conquistar sus objetivos, basados en la tendencia descrita por Verkhosshansky (2002), fundamentada en los avances de la biología teórica, la fisiología de la actividad muscular, la anatomía y morfología funcional, la biomecánica y bioenergética del deporte, la medicina deportiva y la psicología y psicomotricidad del hombre. Esta requiere procesos a largo plazo ininterrumpidos, con inversión, con apoyo del Estado y del sector privado. Platonov (2001) plantea que se pueden establecer tres tipos de deporte moderno con diferente orientación y criterios de eficacia: 1) deporte olímpico; 2) deporte de masas y, 3 ) deporte profesional, cada uno con objetivos que el autor deja claros y delimita sus alcances.
Para el contexto colombiano, la ley 181 de 1995, en el Titulo IV del deporte, capítulo I en las definiciones y clasificaciones, artículo 15, define: "El deporte en general es la específica conducta humana caracterizada por una actitud lúdica y de afán competitivo de comprobación o desafío expresada mediante el ejercicio corporal y mental, dentro de disciplinas y normas preestablecidas orientadas a generar valores morales, cívicos y sociales". En esta misma ley, en el artículo 16, se delimitan las manifestaciones del deporte nacional y será el soporte para la formulación de políticas de promoción y de fomento.

El deporte formativo es aquel que tiene como finalidad contribuir al desarrollo integral del individuo con prácticas multivariadas, abarcando los procesos de iniciación, de fundamentación y de perfeccionamiento deportivo y que tienen lugar, tanto en los programas del sector educativo formal y no formal como en los programas desescolarizados, de las escuelas de formación deportiva y semejantes. La ley delimita, también, otras manifestaciones que deben ser resultado del proceso del deporte formativo, como lo es el deporte comunitario, universitario, asociado, competitivo, de alto rendimiento, aficionado y profesional. Con esta ley, el deporte nacional tiene la posibilidad de organizar las acciones para su fomento, pero si a estos elementos le sumamos los lineamientos curriculares para la educación física y la ley 934 de 2004, que dicta orientaciones y disposiciones para la creación de centros de educación física, de iniciación y de formación deportiva, además de un sinnúmero de condiciones para la práctica sistémica, organizada, sostenida en el tiempo con propósitos educativos, sociales, culturales y de salud, entre otros, (Peralta, 2006), se debe pensar que, efectivamente, existe lo indispensable para desarrollar y mantener una cultura deportiva en el país, pero que no es suficiente para garantizar un desarrollo integral del individuo

En la última década, organizaciones mundiales, como Panamerican Health Organization (2005), Alianza Mundial de los Deportes, UNESCO, OMS (ONU, 2010), CDC de Atlanta (2010) y ACSM (2009), entre otras, presentaron recomendaciones concretas y propuestas para la práctica continua del deporte, desde el pre-escolar hasta la vida adulta. Las recomendación fueron actividad física y práctica de deportes, por lo menos tres veces a la semana, por treinta minutos de duración y con intensidad de moderada a vigorosa, para obtener beneficios para la salud y el desarrollo y social. Se han realizado también varios congresos en el país, que convocan a comunidades científicas y académicas a reflexionar sobre el fenómeno deportivo, buscando hacer eco en los dirigentes y políticos responsables sobre la importancia del tema, para no solo el éxito deportivo del país sino para construir una sociedad, donde el hombre se reconozca como un fundamento y elemento de su entorno. 
Al conceptuar un poco más profundo sobre la iniciación y la formación deportiva, se debe comprender el énfasis en el concepto de proceso, que varios autores analizan. Blázquez (2010) caracteriza la iniciación y la formación deportiva como un proceso de socialización, un proceso de integración, un proceso de enseñanza-aprendizaje, un proceso de adquisición de capacidades, de habilidades, de destrezas, de conocimientos y de actitudes, un proceso de inclusión y de participación. Para Hernández et al. (2000), el proceso de iniciación deportiva no es el momento del inicio de la práctica deportiva, sino que es el inicio de una acción pedagógica que, teniendo en cuenta las características del niño y sus objetivos, va evolucionando hasta alcanzar el dominio de la especialidad. Rusch \& Weineck (2004) plantean objetivos para la clase de formación deportiva complementaria a clases de educación fisca, procesos de mejora de la salud en general, gusto e interés por el movimiento y la motivación por deportes posterior a la escuela, entre otros.

El proceso de iniciación y de formación deportiva genera diferentes tipos de ventajas para la aprendizaje de la población, físicamente activa y con diversos tipos de valores sociales (Añó, 1997). Como proceso, busca mejorar y especializarse, pero sin perder la óptica que no todos los jóvenes serán deportistas de altos logros (Zhelyazkoz, 2001). Así como la especialización científica, en vía de la profundización, no obstaculiza sino que contribuye a la enseñanza en general, del mismo modo, el deporte de alto rendimiento estimula el deporte de masas, que permite tener referentes de proceso para programas y planes más eficientes, con más contenido motriz intelectual y emocional.

Los procesos en el deporte deben garantizar acciones lógicas, secuenciales y progresivas en busca de bienestar, de integración y de socialización, a todo nivel de las esferas humanas. Si la dirigencia deportiva y los políticos responsables comprenden los beneficios de un proceso, los apoyos, los esfuerzos y los recursos llegarán a la base de la pirámide, donde es el inicio de todo: la infancia y los niños colombianos. Ellos requieren atención de los entes estales para iniciar y no interrumpir procesos que los hagan mejores ciudadanos, con una alta autoestima por su cuerpo y por su salud, que respeten el medio ambiente y, por supuesto, que sea la semilla de deportistas de alto rendimiento (Ramos \& Taborda, 2010).

La evidencia científica y las observaciones clínicas, que en la actualidad se plantean y que están disponibles sobre los beneficios para la salud en los procesos de actividad física, del ejercicio y del deporte formativo, ha comprobado mejora en la formación de la estructura ósea, una óptima composición corporal, una excelente respuesta metabólica y un menor número de lesiones. Se ha registrado, desde hace varias dé- cadas, por diferentes autores, estos beneficios, así como los riesgos del sedentarismo, los malos hábitos nutricionales y el consumo de tabaco, que aumentan de manera dramática el número enfermedades crónicas degenerativas, la obesidad y los riesgos cardiovasculares (Pate et al. 1995; Shephard et al. 1991; Caspersen et al. 1985; Pollock et al. 1990; Slattery, 1996; Nacleiro, 2011). Es claro que el movimiento, el ejercicio y el deporte orientado hacia la salud física, social y ambiental es la inversión más rentable para cualquier país.

Esta idea es muy importante en el concepto de iniciación y de formación deportiva, de proceso, de continuidad, que con la cercanía de los Juegos Olímpicos, el país debe pensar en la evaluación de sus procesos que se llevaron a cabo para llegar a este evento: ccuántos niños comenzaron el proceso?, ¿cuántos iniciaron procesos de especialización deportiva?, ¿cuántos llegaron a altos logros?, ¿cuál es la inversión en tiempo, en recursos y en apoyo de las diferentes ciencias aplicadas?, ¿existe registro de marcas por edad?, ¿existen tablas en todas disciplinas deportivas de evaluaciones específicas para la selección de talentos que correspondan a las características nacionales?, cicuál es la formación y la experiencia de los entrenadores?, cंcuál es el apoyo para la formación de entrenadores?

En repetidas ocasiones en el deporte nacional se piensa primero en la clasificación y después en el proceso, en la planeación y en la planificación de futuro. La medalla es el objetivo a alcanzar, pero poco se prevé el mantener el logro o el realizar un procedimiento de desentrenamiento deportivo. La planificación deportiva para Vasconcelos (2005) es anticipar, prever una secuencia lógica y coherente del desarrollo de tareas que nos llevarán a alcanzar objetivos, previamente definidos. Se comprende la planificación como el proceso que define métodos y acciones desde la iniciación al deporte y su rendimiento, sin descuidar al deportista en su desentrenamiento (García et al. 1996; Bompa, 2000; García-Verdugo, 2000; Weineck, 2005; Baechle E Earle, 2007).

En la actualidad, los avances en tecnología, la sistematización y las comunicaciones, más los instrumentos con que se mide en el deporte, permiten e invitan a profesionales a llevar a cabo investigaciones en aspectos propios del deporte: físicos, técnicos y tácticos, además de las ciencias aplicadas y sociales (Heinemann, 2005; Hohmann et al. 2005); por otra parte, se suma el aumento de tiempo de ocio, el incremento de estilos de vida sedentarios y un modelo de educación que desconoce la cultura física, de manera que construir, aplicar y mantener la cultura deportiva se convierta en un verdadero reto para el país, en materia salud pública y social.

El entrenador o el docente de deportes, en los diferentes estadios del proceso, pero fundamentalmente en la iniciación 
y en la formación deportiva, debe contar con una capacidad profesional muy alta; sus estudios deben abarcar áreas de la sicopedagogía, ciencias básicas, ciencias aplicadas, recursos informáticos y tecnológicos aplicados al deporte y, por supuesto, una praxis argumentada. Martens (2002) considera también, que el éxito del entrenador radica en su filosofía, entendida como la capacidad de aplicar las creencias y los principios a las acciones que guían su tarea profesional, en otras palabras, el entrenador debe ser un profesional altamente calificado y en constante formación. Es inadmisible que exista un alto número de entrenadores que tienen niños a su cargo sin capacitación y, peor aun, dirigentes deportivos que desconocen aspectos teóricos y prácticos del deporte. Navarro (2004) reflexiona sobre esta problemática, que se convierte en un peligro para los niños y el desarrollo del deporte.

Que buen momento para pensar y para reflexionar en el deporte formativo y proyectar la selección de talentos, que oriente a niños y a jóvenes en la práctica divertida del deporte para toda la vida, de modo que quienes cumplan con criterios definidos, claramente por la disciplina deportiva, inicien procesos de especialización, basados en diversas características, como las descritas por Martin et al. (2004): la predisposición o potencial genético, condiciones del entrenamiento en el entorno, el margen de tiempo que se requiere de manera individual para alcanzar la forma deportiva que, combinadas con características intra e interindividuales, conforman el talento deportivo.

En la selección del talento deportivo no se debe desconocer lo planteado por García et al. (2003), sobre los aspectos metodológicos en su elección, basados en rasgos individuales, en componentes cognitivos, en orientaciones sobre el logro y los ámbitos socio-culturales, que deben caracterizar cada disciplina deportiva, desde la misma experiencia y que deben involucrar otras variables, que la práctica propia del deporte dicte.

La articulación de un proceso de iniciación y de formación deportiva sumado la selección de talentos, se constituye en modelo fundamental que debe construir todas las federaciones y ligas deportivas, que pretenda contribuir con la formación de mejores ciudadanos y deportistas de élite, sustentados en las ciencias aplicadas (Domínguez \& Espeso, 2003).

De acuerdo con Leiva (2010), los modelos se caracterizan por la construcción de "modelos" de objeto investigado y servirse de éste para la obtención de una reducción, la cual, se puede reproducir por analogía. Las bases científicas, metodologías, logísticas y financiera para la construcción de modelos comprometen a profesionales altamente calificados, con conocimiento de las particularidades del deporte nacional.
La responsabilidad de la sociedad, de los padres, de los educadores de los entrenadores y de los dirigentes deportivos, con la elaboración de modelos, debe estar de acuerdo con los reportes de las comunidades científicas y académicas, en cuanto a los beneficios y los posibles impactos de la práctica deportiva sobre el organismo y aspecto socio-culturales. Indican Thiebauld \& Sprumont (2009), que en nuestra sociedad de opulencia y consumista, el deporte y el niño están implicados en relaciones complejas, así que no puede ser el líder del proceso de iniciación deportiva una persona sin formación en aéreas de la pedagogía, que adolezca de conocimientos profundos en crecimiento, en desarrollo y en maduración y en ciencias aplicadas; solo con excelentes profesionales, se garantizará el bienestar de los niños y la selección de futuros deportistas.

El trabajo es fuerte y no se puede obviar justo cuando el resultado de los procesos entra en evaluación con las justas olímpicas. Es importante la cualificación constante y la certificación de docentes entrenadores y de líderes deportivos. Los recursos para el deporte, se deben garantizar bajo el concepto del proceso y no se deben interrumpir por cambios de los políticos a cargo o burocracia, que no conoce ni se informa antes de trabajar en el sector. Federaciones y ligas deben construir, apoyados por las instituciones rectoras, las caracterizaciones de sus disciplinas deportivas, los procesos de iniciación, de formación y de selección de talentos y elaborar modelos que premien la flexibilidad propia de los seres humanos.

Es momento de apuntalar las nacientes propuestas del Ministerio de Educación con las Jornadas Escolares Complementarias y el programa Supérate (2011), que apoyadas por Coldeportes busca desarrollar las competencias deportivas, generar hábitos y estilos de vida saludables, para que sean un real proceso de educación y de formación y no terminen como un proyecto más donde se invierten recursos y no generan resultados tangibles. Son propuestas que se deben articular a la ley 934 de 2004, orientadas por el Ministerio y Coldeprotes, en lo que respecta a procesos de evaluación y de diagnóstico, educación motora y físico, iniciación y formación deportiva y selección de talentos deportivos, que nutran las clubes y ligas deportivas del país, acompañadas por veeduría ciudadana y apoyo de expertos, para construir la cultura física que requiere el país.

Agradecimientos: El autor expresa sus agradecimientos a los evaluadores de esta revisión. Financiación: Esta revisión fue apoyada por la Universidad de Ciencias Aplicadas y Ambientales U.D.C.A. Conflicto de intereses: El autor declara que no existe ningún conflicto de intereses que pongan en riesgo la validez de la revisión presenta. 


\section{BIBLIOGRAFÍA}

1. ACOSTA, E. 2008. Educación física y desarrollo Humano, aproximación conceptual de las líneas de investigación. Ed. Universidad Libre Bogotá. 79p.

2. ACSM's. 2009 American College of Sports Medicine Guidelines for Exercise Testing and Prescription. 188p.

3. AÑÓ, V. 1997. Planificación y Organización del Entrenamiento Juvenil. Ed. Gymnos. Madrid. 24p.

4. BAECHLE, T.; EARLE, R. 2007. Principios del entrenamiento de la fuerza y del acondicionamiento físico. Ed. Médica Panamericana. Madrid. 515p.

5. BLÁZQUEZ, D. 2010. La iniciación deportiva y el deporte escolar. Ed. INDE Barcelona. 24p.

6. BOMPA, T.O. 2000. Periodización del entrenamiento deportivo. Ed. Paidotribo. $1^{\text {a }}$ ed. Barcelona. 209p.

7. CAGIGAL, J. 1975. El deporte en la sociedad actual. Madrid: Prensa Española y Magisterio Español. (España). 157p.

8. CAGIGAL, J. 1990. Deporte y agresión. Ed. Alianza editorial, concejo superior de deporte Madrid. 73p.

9. CASPERSEN, C.J.; POWELL, K.E.; CHRISTENSON, G.M. 1985. Physical activity, exercise, and physical fitness: Definitions and distinctions for health-related research. Public Health Reports. 100(2):126-131.

10. Centers for Disease Control and Preventio CDC. 2010. The Association Between School-Based Physical Activity, Including Physical Education, and Academic Performance. Disponible desde Internet en: http:// www.cdc.gov/healthyyouth/health_and_academics/ pdf/pa-pe_paper.pdf (con acceso 01/12/2011).

11. COMITÉ OLIMPICO INTERNACIONAL COI. 2010. Disponible desde Internet en: http://www.olympic.org/ educators (con acceso 01/12/2011).

12. CONTECHA, L.F. 1999. La Educación Física y el Deporte en Colombia. Una historia. Disponible desde Internet en: www.efdeportes.com (con acceso 1/12/2011).

13. DECRETO 2743 de 1968, por el cual se crea el Concejo Nacional de la Juventud y el Deporte (COLDEPORTES). Disponible en internet en: http://www.indercas. gov.co/leyes/09 (con acceso 01/12/2011).
14. DIEM, C. 1966. Historia de los deportes. Vol. I. Ed. Luis de Caralt, Editor Barcelona. 479p.

15. DOMÍNGUEZ, P.; ESPESO, E. 2003. Bases fisiológicas del entrenamiento de la fuerza con niños y adolescentes. Rev. Int. Med. Cienc. Act. Fís. Dep. 3(9):6168. Disponible desde Internet en: http://cdeporte. rediris.es/revista/revista9/artfuerza.htm (con acceso 11/10/2012).

16. ELÍAS, N.; DUNNING, E. 1992. Deporte y ocio en el proceso de la civilización. Ed. Fondo Cultura Económica. México. 341p.

17. GARCÍA, J.; NAVARRO, M.; RUIZ, J. 1996. Planificación del entrenamiento deportivo. ed. Gymnos Madrid. 9p.

18. GARCÍA-VERDUGO, M. 2000. Principios del autoentrenamiento. Ed. Gymnos. Madrid. 90p.

19. GARCÍA, J.; CAMPOS, J.; LUIZAR, P.; ABELLA, C. 2003. El talento deportivo formación de elites deportivas. Ed. Gymnos. Madrid. 20p.

20. HEINEMANN, K. 2005. Introducción a la metodología de la investigación empírica. El ejemplo de las ciencias del deporte Ed. Paidotribo. Barcelona. 10p.

21. HERNÁNDEZ, M.; CASTRO, U.; CRUZ, H.; GIL, G.; GUZMÁN, G.; QUIROGA, M.; RODRÍGUEZ, P. 2000. La iniciación a los deportes desde su estructura y dinámica. Ed. INDE. Barcelona. 11p.

22. HERNÁNDEZ, R.; FERNÁNDEZ, C; BAPTISTA, P. 2006. Metodología de la investigación. Ed. Mc. Graw Hill. México. 216p.

23. HERNÁNDEZ, R.; FERNÁNDEZ, C; BAPTISTA, P. 2007. Fundamentos de metodología de la investigación. Ed. Mc. Graw Hill. México. p.59 142.

24. HOHMANN, A.; LAMES, M.; LETZELTER, M. 2005. Introducción a la Ciencia del Entrenamiento. Ed. Paidotribo. Barcelona. 23p.

25. KOCH, K. 1981. Hacia una ciencia del deporte. Ed. Kapelusz Buenos Aires. 95p.

26. LEIVA, J. 2010. Selección y orientación de talentos deportivos Ed. Kinesis y Ed. Universidad del Valle. Armenia. 115p. 
27. LEY 181 de enero 18 de 1995, por el cual se dictan disposiciones para el fomento del deporte, la recreación, el aprovechamiento del tiempo libre y la Educación Física y se crea el Sistema Nacional del Deporte. Disponible desde Internet en: http://www. mineducacion.gov.co/1621/articles-85919_archivo_ pdf.pdf (con acceso 02/12/2011).

28. LEY 934 de 2004, por la cual se oficializa la Política de Desarrollo Nacional de la Educación Física y se dictan otras disposiciones. Disponible desde Internet en: http://www.secretariasenado.gov.co/senado/basedoc/ley/2004 (con acceso 02/12/2011).

29. MARTENS, R. 2002. El entrenador de Éxito. Ed. Paidotribo. Barcelona. p.11.

30. MARTIN, D.; NICOLAUS, J.; OSTROSWSKI, C.; ROST, K. 2004. Metodología del Entrenamiento Infantil y Juvenil. Ed. Paidotrivo Barcelona. 169p.

31. MINISTERIO DE EDUCACION NACIONAL. s/f. Disponible desde Internet en: http://www.mineducacion. gov.co/cvn/1665/w3-article-279856.html (con acceso $02 / 11 / 2011)$.

32. NACLEIRO, F. 2011. Entrenamiento deportivo, fundamentos y aplicación en diferentes deportes. Ed. Médica panamericana, Madrid. 399p.

33. NAVARRO, F. 2004 Entrenamiento adaptado a los jóvenes. Rev. Ed. 335:61-80.

34. ONU Asamblea general Naciones Unidas. 2010. El deporte como medio de promover el desarrollo y la paz: progreso y perspectivas. Disponible desde Internet en http://daccess-dds-ny.un.org/doc/UNDOC/GEN/ N07/504/06/PDF/N0750406.pdf?OpenElement (con acceso1/12/2011).

35. PANAMERICAN HEALTH ORGANIZATION. 2005. IMCI Community Component: guide for teachers and theirs schools. Washington, D.C: PAHO. 52p.

36. PARLEBAS, P. 2001. Juegos Deporte y Sociedad. Léxico de praxiología motriz Ed. Paidotribo. Barcelona. 271p.

37. PATE, R.R.; PRATT, M.; BLAIR, S.N.; HASKELL, W.L.; MACERA, C.A.; BOUCHARD, C.; BUCHNER, D.; ETTINGER, W.; HEATH, G.W.; KING, A.C.; KRISKA, A.; S. LEON, A.S.; MARCUS, B.H.; MORRIS, J.; PAFFENBARGER Jr, R.S.; PATRICK, K.; POLLOCK,
M.L.; JAMES M. RIPPE, J.M.; SALLIS, J.; WILMORE, J.H. 1995. Physical Activity and Public Health: A Recommendation from the Centers for Disease Control and Prevention and the American College of Sports Medicine. JAMA. 273(5):402-407.

38. PERALTA, H. 2006. Centros de educación física, escuelas de iniciación y formación deportiva. Una alternativa en cultura física para Colombia a través de experiencias en el departamento de Cundinamarca. Ed. Átropos Bogotá. p.17-24.

39. PLATONOV, V. 2001. Teoría general de entrenamiento olímpico. Ed. Paidotribo. Barcelona. 10p.

40. POLLOCK, M.L.; WILMORE, J.H.; FOX III, S.M. 1990. Exercise in Health and Disease: Evaluation and Prescription for Prevention and Rehabilitation. 2nd ed. Philadelphia: W.B. Saunders Co. p.100-110; p.371484.

41. RAMOS, S.; TABORDA, J. 2010. Orientaciones para la planificación del entrenamiento con niños. Ed. Fís. Dep. 65:45-52. Disponible desde Internet en: http:// www.articulos-apunts.edittec.com/65/es/065_045052ES.pdf (con acceso 11/10/2012)..

42. RODRÍGUEZ, J. 2000. Historia del Deporte. Ed. Inde publicaciones. Zaragoza. 170p.

43. RUSCH, H.; WEINECK, J. 2004. Entrenamiento y Práctica Deportiva Escolar. Ed. Paidotrivo. Barcelona. 60p.

44. SHEPHARD, R.J.; STEPHENS, T.; SUTTON, J.R.; MCPHERSON, B.D. (Eds.). 1991. Exercise Fitness, and health: A Consensus of Current Knowledge. Champaign, IL: Human Kinetics Books. p.155-163.

45. SLATTERY, M.L. 1996. How much physical activity do we need to maintain health and prevent disease? $\mathrm{Di}$ fferent disease-Different mechanism. Res. Quart. Exerc. Sport. 67(2): 209-212.

46. THIEBAULD, C.; SPRUMONT, P. 2009. El niño y el deporte. Tratado de Medicina del Deporte Infantil Ed. Inde Publ. Zaragoza. 629p.

47. VASCONCELOS, A. 2005. Planificación y organización del entrenamiento deportivo Ed. Paidotribo. Barcelona. 19p.

48. VELÁSQUEZ, R. 2001 El deporte moderno. Consideraciones acerca de su génesis y de la evolución de su 
significado y funciones sociales. Ed. Fís. Deport. Rev. Digital. Buenos Aires. 7(36) - $^{\circ} 36$ Disponible desde Internet en: http://www.efdeportes.com/efd36/deporte.htm (con acceso 01/12/2011).

49. VERKHOSSHANSKY, Y. 2002. Teoría y metodología del entrenamiento deportivo. Ed. Paidotribo. Barcelona. $15 p$.
50. WEINECK, J. 2005. Entrenamiento Total. Ed. Paidotribo. Barcelona. 45p.

51. ZHELYAZKOZ, T. 2001. Bases del Entrenamiento Deportivo. Ed. Paidotribo Barcelona. 19p.

Recibido: Enero 18 de 2012

Aceptado: Marzo 26 de 2012 